\title{
A professional identity and resilience of students
}

\author{
Nadezhda Zubareva ${ }^{1, *}$, Valeriia Kapustina $^{1}$, Marina Matyushina ${ }^{1}$ \\ ${ }^{1}$ Novosibirsk State Technical University, Department of psychology and pedagogy, 630073, Novosibirsk, Russia
}

\begin{abstract}
The article is devoted to the theoretical analysis of resilience as a psychological concept and its connection to professional identity. The article includes the results of pilot studies of professional identity and creativity, resilience of students in a context of professional resilience. The sample consisted of students of Novosibirsk State Technical University ( 80 and 51 participants respectively). Methods of the research were theoretical analysis and psychological tests. It was found that resilience is an integrative dynamic characteristic, which includes hardiness as an ability to cope with adverse events, adaptation as an ability to change in different situations, value and semantic components as a way of thinking on the experience lived through. The professional resilience is a complicated system of personal (internal and existential) and organizational resources, which are necessary to sustain well-being of a professional. It was found that most students have medium and high levels of resilience. Students with professional identity moratorium in comparison with students with achieved professional identity want to be more intuitive in a making decision and suggest consequences of decisions. Also these students want to think more creatively, to create a humorous product and not to be afraid to be funny in some situations of life.
\end{abstract}

\section{Introduction}

The rapid development of technology forces people to quickly adapt to innovations in society and in the professional environment. Robotics, virtual and augmented reality, artificial intelligence, chat bots create a situation, in which, on the one hand, a person needs to quickly learn to work in new conditions, on the other hand - to compete in the professional field not with people, but with technology. Progress and humancreated benefits have reduced the need for survival and adjustment at the physical level, but at the level of the psyche the pressure continues to grow. It makes important to study the influence of changing living conditions on the resilience and professional identity of today's youth.

\section{An overview}

\subsection{Methods}

Methods of the research were:

- theoretical analysis of scientific literature;

- psychological tests («Adult Resilience Measure Michael Ungar» ARM-R by M. Ungar (translation of V.A. Kapustina); "Adult resilience" by A.V. Mahnach; "Test of professional identity status" by A.A. Azbel, A.G. Gretsov; test of creativity by N.F. Vishnjakova).

\subsection{Hypotheses}

1. The professional identity of students and their resilience are connected.

2. The creativity of students differs in groups with a different level of professional identity.

\subsection{Professional resilience as a psychological concept}

There's no common opinion about resilience as a psychological phenomenon: it explores a personal trait, ability, dynamic process, so we consider how this concept could be defined.

G. Bonanno believes that resilience is a response to adverse events and it is an attribute of healthy functioning. R. Yehuda adds that resilience is not just the ability to recover from exposure to a certain stressor; it allows people to learn a lesson from adverse experiences and advances the development of a person [1].

N.T. Selezneva, N.V. Rublenko define resilience as an integrative dynamic feature, represented by value orientations, striving for self-development and motivation of achievements, abilities to function and develop successfully [2].

E.V. Kuftjak attributes to resilient people a sense of the meaningfulness of life, a positive attitude towards themselves and others, a tendency to change, the ability to solve problems, to adapt and control their lives [3].

L. Liu, X. Xu, N. Xu, L. Wang describe resilience as a psychological ability of a person to recover from negative situations. They've found that resilience could decrease the association between disease activity and mental component health related quality of life [4].

\footnotetext{
*Corresponding author: ns.zubareva@mail.ru
} 
M.C. Johnston et al. revealed that resilience is a dynamic process (not a personal trait) and it involves maintaining healthy levels of functioning following adversity [5].

E.V. Lapkina argues that in addition to coping with stress, resilience is a part of a system of life meanings and attitudes of an individual, determining the specifics of a response to stress. Resilience affects the quality of life of an individual during periods of stability and during difficult life periods. Person with developed resilience percepts even traumatic events as a life experience that is not capable of knocking them out [6].

This definition is similar to understanding the resilience of Grant and Kinman. They describe resilience as "a positive construct which enables individuals to overcome stressors or withstand negative life events and, not only recover from such experiences, but also find personal meaning in them" [7].

G.I. Borisov and A.A. Pecherkina consider that resilience is a personal resource of professional development, so it can help person to cope with negative conditions in professional activity by adapting to changes [8].

As we can see in all these definitions, resilience is an integrative dynamic characteristic, which includes hardiness as an ability to cope with adverse events, adaptation as an ability to change in different situations, value and semantic components as a way of thinking on the experience lived through.

It seems to be important to review the resilience in a context of professional environment.

S.N. Vasil'eva and E.A. Rylskaja define professional resilience as a personal ability to successful adaptation, surviving, self-development by individual resources, competency, behavioral strategies in a structural and regulatory environment of an organization [9]. These authors identified three concepts of professional resilience: ability, organization and personality, through which they revealed the skills and personal features, which are necessary for the successful functioning of a person in a professional environment. For example, the ability to cope with emerging difficulties, recover after it, adapt to changes, to society, develop, learn, use resources to cope with stress, successfully solve professional tasks, find ways to live in a profession, maintain personal integrity in the structure of the organization, grow in it, to know and observe the norms of joint activity, to be subjectively satisfied with the work [9].

A.R. Rosenberg describes the professional resilience as "the process of harnessing the resources we need to sustain well-being" [10]. According to A.R. Rosenberg, it's important to develop three types of resources to exist successfully in an organization and to prevent an emotional burn-out [10]:

- external resources (exchanges of the experience with colleagues, professional relations, supportiveness);

- internal resources include adaptive personal traits (persistence, optimism, sense of humor) and skills of stress and time-management (goal-setting, constructive response to criticism, achieving work-life balance);
- existential resources - reflection and selfawareness, understanding the motive of choosing a profession and their priorities.

O.A. Pliushcheva also calls three levels of professional resilience, similar in some parameters to the concept of A.R. Rosenberg (the individual level can be compared with the internal resources and the group with the external ones) [11]:

- Individual level includes personal and professional experience, mental and personal skills and features, related to the requirements of the profession.

- Group level - communication, support and assistance of colleagues, praise, exchange of the experience.

- Organizational level - workflow, conditions and mode of work, organization of the workplace, etc.

We suppose that professional identity could be included to existential resources of the professional resilience.

This stage of analysis allows us to conclude that the professional resilience is a complicated system of personal (internal and existential) and organizational resources, which are necessary to sustain well-being of a professional.

\subsection{Professional identity}

Consider the existing ideas about the content of professional identity.

L.B. Schneider understands professional identity as the result of professional self-determination, personalization and self-organization. It is manifested in self-awareness as a representative of a certain profession and professional community, a certain degree of identification and differentiation of oneself with Deed and Others. Also it is manifested in three selfdescriptions of Self: cognitive, emotional and behavioral $[12,13]$.

M.D. Holden focuses on the ethical and value side of professional identity. This author understands professional identity as an active process of deepening personal commitment to the values of the profession and the development of internal standards of professional activity [14].

According to Yu. Povarenkov, professional identity is the criterion of professional development, testifying to the degree of a person's acceptance of a certain professional activity as a way of self-realization; systems of professional norms and values, and professional self [15].

S.I. Kudinov, Yu.V. Vladimirova consider that professional identity is a significant factor in a personal psychological well-being, because it provides a "psychoecological balance", i.e. a sense of the stability of the world and self-reliance [16].

C. Tang, S.E. Naumann do not distinguish professional identity as such; however, they indicate two specific types of identity: command and competence. The command identity means that a person belongs not only to a certain social group, but to a particular organization, so command identity reflects the degree of 
acceptance of an organizational culture and the vision of personal future in a connection with the future of the organization. Competency identity is more associated with the position that a person holds, as well as with his professional experience and self-image in the course of this position. This kind of identity is positively correlated with creativity [17].

E.P. Ermolaeva supposes that professional identity is a product of long-term personal and professional development, which develops only at high levels of mastering a profession and acts as a sustainable coordination of the main elements of the professional process (coordination of Actual and Ideal professional Self) [18].

I.Yu. Khamitova believes that the process of professional personal identity requires at least four years [19].

Measuring of a professional identity seems to be complicated.

A.A. Azbel and A.G. Gretsov have based their representations about professional identity's levels on J. Marcia's identity status theory [20]:

1) diffused professional identity: life choices have not been made, there are no clear ideas about a career;

2) foreclosed professional identity: a person has formed ideas about his professional future, but it is not the result of an independent choice, it's a choice imposed from outside (for example, by parents);

3) professional identity moratorium: a person is aware of the problem of choosing a profession and is in the process of solving it, but the most suitable option has not yet been determined;

4) achieved professional identity: professional plans are defined, it is the result of a meaningful independent decision.

M.D. Merino, J. Privado suppose that Resilience, Creativity, Autonomy, Self-Esteem, Purpose in life, Enjoyment, Optimism, Curiosity, Humor, Environmental mastery can be merged to the general construct called Positive Psychological Functioning, because these psychological resources are associated with well-being, ability to manage and cope with life [21].

At the same time professional identity contributes to the successful professional personal adaptation and acts as a leading factor in a career [22].

J. Pears and C. Morrison suppose that teachers' identity formation and resilience are connected [23].

Also S. Rajan-Rankin has found that self-identity of social work students is "an important foregrounding factor in the management of emotion and changes in their self-hood are pivotal to their approach towards emotionality and capacity for resilience" [24].

\section{Results and discussion}

The empirical study of professional identity and resilience of students was held by authors in Novosibirsk State Technical University.

The object of study is a professional identity and resilience of students.
The purpose of study is to find out characteristics of professional identity and resilience of students.

\subsection{The results of an empirical study of a professional identity of students}

The conditions of the modern world dictate special requirements for graduates to their abilities to independently make decisions regarding their professional path, to develop and implement nonstandard and fundamentally new ideas, to apply flexibility in solving professional problems. All these characteristics are included in the concept of creativity. That's why we decided to study a professional identity in a correlation with self-esteem of creativity.

The sample of the study consisted of 80 students of Novosibirsk State Technical University and Siberian State University of Railway Transport (35 males, 45 females, age - 17-22 years old).

The most identity status in this sample was professional identity moratorium, (60\% of respondents): the students have decided what profession they want to get, but they do not see their professional future, they do not have clear professional goals. These results confirm the data of the Federal Labor and Employment Service of Russia, according to which only $27 \%$ of university graduates work on the received specialty in 2017. Probably, this may be the result of an unformed professional identity: university graduates enter the labor market with professional knowledge, but without a clear understanding of the content of the future profession and the vision of themselves and their future in this professional field.

We have found significant differences between groups with achieved and diffused identity status (table 1).

Table 1. - Significant differences between groups with achieved and diffused identity status, $\mathrm{p} \leq 0.05$

\begin{tabular}{|l|l|l|l|}
\hline Scale & $\begin{array}{l}\text { U-meaning } \\
\text { (Mann- } \\
\text { Whitney U- } \\
\text { test) }\end{array}$ & $\begin{array}{l}\text { Achieved } \\
\text { profession } \\
\text { al identity } \\
\text { status } \\
\text { (N=19) }\end{array}$ & $\begin{array}{l}\text { Diffuse } \\
\text { d } \\
\text { identity } \\
\text { status } \\
\text { (N=12) }\end{array}$ \\
\hline $\begin{array}{l}\text { Originality } \\
\text { (Actual Self) }\end{array}$ & 89.5 & 6.2 & 5.7 \\
\hline $\begin{array}{l}\text { Intuition } \\
\text { (Actual Self)) }\end{array}$ & 56.5 & 6.2 & 5.2 \\
\hline $\begin{array}{l}\text { Humor } \\
\text { (Ideal Self) }\end{array}$ & 109.5 & 6.8 & 6.75 \\
\hline
\end{tabular}

As we can see in the table 1, students with achieved professional identity status have more expressed selfesteem in originality, intuition and humor (Actual self), than students with diffused identity status. It means that students with achieved professional identity status (who have made their professional choice consciously and independently, having an idea of their professional future) are more than students with diffused identity status, assess themselves as original, capable of 
generating non-sample and non-standard ideas and solutions, tend to rely on intuition, while would like to be easy to relate to the situation, create a humorous product and not be afraid to be funny in some situations of life.

Table 2. - Significant differences between groups with achieved identity status and identity moratorium, $\mathrm{p} \leq 0.05$

\begin{tabular}{|l|c|c|c|}
\hline Scale & $\begin{array}{l}\text { U-meaning } \\
\text { (Mann- } \\
\text { Whitney U- } \\
\text { test) }\end{array}$ & $\begin{array}{l}\text { Achieved } \\
\text { profession } \\
\text { al identity } \\
\text { status } \\
\text { (N=19) }\end{array}$ & $\begin{array}{l}\text { Profession } \\
\text { al identity } \\
\text { moratoriu } \\
\text { m (N=46) }\end{array}$ \\
\hline $\begin{array}{l}\text { Creative } \\
\text { thinking } \\
\text { (Ideal Self) }\end{array}$ & 275 & 6.2 & 7.5 \\
\hline $\begin{array}{l}\text { Intuition } \\
\text { (Ideal Self) }\end{array}$ & 239 & 5.7 & 7 \\
\hline $\begin{array}{l}\text { Humor } \\
\text { (Ideal Self) }\end{array}$ & 254 & 6.9 & 7.9 \\
\hline
\end{tabular}

As we can see in the table 2, Creative thinking (Ideal Self), Intuition (Ideal Self) and Humor (Ideal Self) are more expressed in group with professional identity moratorium.

It means, that students with professional identity moratorium in comparison with the students with achieved professional identity want to be more intuitive in a making decision and suggest consequences of decisions, to think more creatively, to create a humorous product and not be afraid to be funny in some situations of life.

The correlative analysis with using of Rs-criterium of Spearman found following correlations between components of professional identity and creativity:

- Positive correlation between a foreclosed professional identity and an intuition (Actual Self) (Rs=0.36, $\mathrm{p}<0.001)$. It means that students the professional choice of whom was made by an influence of a referent group instead of their own interests and wishes are intuitive in a making decision.

- Positive correlation between a professional identity moratorium and a humor (Ideal Self) $(\mathrm{Rs}=0.32, \mathrm{p}<0.01)$ : students with a professional identity moratorium want to be able to joke, to treat with humor the situation, to create new jokes.

- Positive correlation between an achieved professional identity and originality (Actual Self) $(\mathrm{Rs}=0.29, \mathrm{p}<0.01)$. Students with achieved professional identity percept themselves as people with original and creative way of thinking, able to work in unusual conditions and to face difficulties. Perhaps, the presence of a professional goal, the awareness of oneself as a representative of a certain profession, the vision of one's professional future provide students with a favorable environment for the actualization of their creative potential.

These results made us to suppose that professional resilience is not developed in students, so we decided to conduct a pilot study to check this hypothesis.

\subsection{The results of a pilot study of a resilience of students}

There're no psychological tests of professional resilience, that's why we used 2 tests of resilience: Adult Resilience Measure (ARM-R) by M. Ungar and Adult resilience test by A.V. Mahnach.

The first test has two scales: personal and relational resilience, the second - six scales: self-efficacy, perseverance, internal locus of control, coping and adaptation, spirituality, family relationships.

According to our understanding of professional resilience we decided to measure it by scales "personal resilience" (ARM-R, M. Ungar), "self-efficiency", "perseverance", "internal locus of control" and "coping and adaption".

The sample of the study consisted of 51 students of Novosibirsk State Technical University (28 males, 23 females, age - 19-21 years old).

The research of students' resilience by ARM-R showed that $76 \%$ of the respondents had a high level of resilience, $24 \%$ had a medium level, and a low level was not detected. According to the test of A.V. Makhnach, $23 \%$ of the subjects had a high level of resilience, $77 \%$ had a medium level, and a low level was not found.

Scales of resilience of both psychological tests have a correlation ( $R s=0.44, p<0.01)$, so it means, these tests use a common understanding of a resilience as a psychological construct.

According to the averaged results of the ARM-R test, students have a high level of personal resilience and relational resilience, related to the important relationship with parents or with the main caregiver, partner, or family.

According to the averaged resuls for individual scales of the Machnach's test of adult resilience, it was found the high level on the "self-efficacy" and "family relationships" scale that confirms the results of an ARMR-test. It means, students of our sample correctly assess their capabilities and believe in their effectiveness, are able to overcome obstacles, cope with stress, have the resources to influence life and professional events, they are able to realize their goals, professional and personal tasks. They actively develop social relationships, including maintaining emotional closeness and positive relationships in the family, which contributes to a better coping with stress.

The low level on the "spirituality" scale indicates that our respondents are not inclined to look for support in the faith, they are not well oriented in the system of spiritual and moral assessments of what is happening outside and inside themselves, in their own and social values.

The medium level on the scales "perseverance", "internal locus of control", "coping and adaptation" means the normal severity of such characteristics as activity in business, plans, undertakings, even when circumstances are above possibilities; self-control, autonomy; adaptation to different situations, finding meaning in active problem solving and positive meaning in difficult situations. 
These results refute our hypothesis about low development of professional resilience of students.

\section{Conclusion}

The ability to make responsible decisions in choosing a profession, to find a personal meaning in professional work, independently create professional life, which in turn requires a high personal and professional resilience, plays an important role for the professional development.

Theoretical analysis let us conclude that resilience is an integrative dynamic characteristic, which includes hardiness as an ability to cope with adverse events, adaptation as an ability to change in different situations, value and semantic components as a way of thinking on the experience lived through. The resilience helps a person to adapt to stress and to withstand and overcome difficulties, including in a professional environment.

In this context the professional resilience could be described as a complicated system of personal (internal and existential) and organizational resources, which are necessary to sustain well-being of a professional.

We suppose that achieved professional identity is a factor which could increase the resilience.

Our pilot studies showed that most students have a high level of a resilience, but students with professional identity moratorium in comparison with the students with achieved professional identity want to be more intuitive in making decision and suggest consequences of decisions, to think more creatively, to create a humorous product and not to be afraid to be funny in some situations of life.

Thus, we need to design and conduct an extensive research of a resilience of students in a context of their professionalization.

The analytical part of the study was conducted with the support of Novosibirsk State Technical University. Project №C19-16.

\section{References}

1. S.M. Southwick, G.A. Bonanno, A.S. Masten, C. Panter-Brick, R. Yehuda, Eur. J. Psychotraumatol, 5, DOI: 10.3402/ejpt.v5.25338 (2014).

2. N.T. Selezneva, N.V. Rublenko, Bul. Of KSPU, 4 (34), 116 (2015).
3. E.V. Kuftiak, Bul. Of KSU, 4, 128 (2016).

4. L. Liu, X. Xu, N. Xu, L. Wang, Retrieved from: https://hqlo.biomedcentral.com/articles/10.1186/s12 955-017-0725-6 (2017).

5. M.C. Johnston, T. Porteous, M.A. Crilly, C.D. Burton, A. Elliott, L. Iversen, et al., Psychosom., 56 (2), 168 (2015).

6. E.V. Lapkina, Bul. Of KSU, 21, 130 (2015).

7. L. Grant, G. Kinman, Soc. Work Ed., 31 (5), 605 (2012).

8. G.I. Borisov, A.A. Pecherkina, Ped. Ed. In Rus, 11, 72 (2015).

9. S.N. Vasil'eva, E.A. Rylskaja, Bul. Of YSPU, 2 (107), 106 (2019).

10. A.R. Rosenberg, Pediatrics, 141 (3), (2018).

11. O.A. Pliushcheva, The current state and prospects of development of labor and organizational psychology, 685 (IP RAS, 2018).

12. L.B. Shneider, World Of Psych., 1, (2001).

13. L.B. Shneider, Personal, gender and professional Identity: theory and diagnosys (MPSI, 2007).

14. M.D. Holden, E.Buck, J. Luk et al., Acad Med., 90 (6), (2015)

15. Yu.P. Povarenkov, Sib. Psych. J., 24, 37 (2006).

16. S.I. Kudinov, Yu.V. Vladimirova, Bul. Of RUDN, 3, (2015).

17. C. Tang, S.E. Naumann, Thinking Skills and Creativity, 21, 123 (2016).

18. E.P. Ermolaeva, Psych. J., 4, 51 (2001).

19. T.V. Maliutina, OHB, 5 (132), 149 (2014).

20. A.A. Azbel, A.G. Gretsov, Psychological tests for high school and students (Piter, 2012)

21. M.D. Merino, J. Privado, An. de psic., 31 (1), 45 (2015).

22. V.A. Shpota, Bul. Of BRGU, 1, (2008).

23. J. Pears, C. Morrison, Austral. J. of Teach. Ed., 36 (1), 49 (2011).

24. S. Rajan-Rankin, The Brit. J. of Soc. Work, 44 (8), 2426 (2014) 